\title{
Internal Fracture Caused by Focusing of Explosive Waves
}

C. C. Hsiao, S. R. Moghe, C. K. Soong and W. L. Yin University of Minnesota, Minneapolis, Minnesota

September 1965

GPO PRICE \$

CFSTI PRICE(S) S

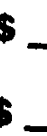

Prepared under

AEC Contract AT(11-1)-532

and

NASA Grant NGR-24-005-070
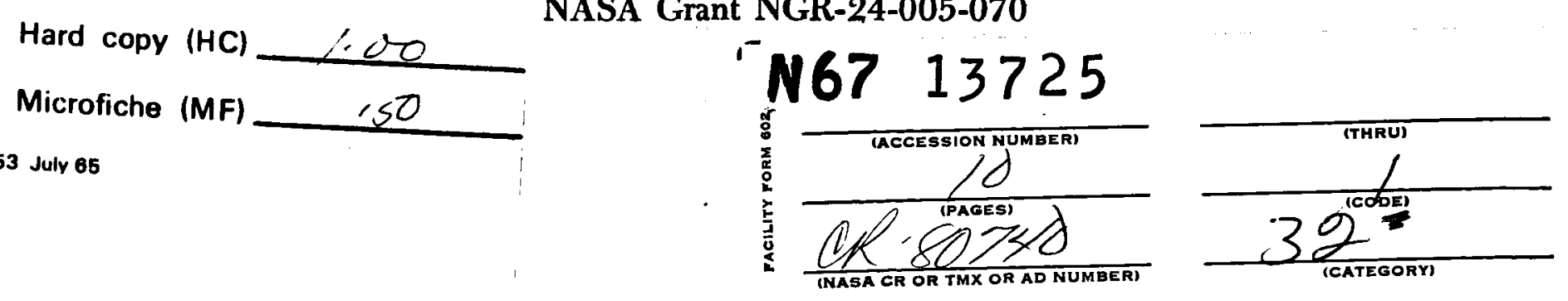
Internal Fracture Caused by

Focusing of Explosive Waves*

C. C. Hsiao, S. R. Moghe, C. K. Soong and W. L. Yin University of Minnesota, Minneapolis, Minnesota

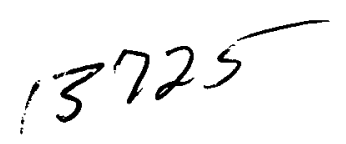

Experimental success in reflecting and focusing explosive waves and thus fracturing the neighborhood of a focus in a prolate spheroid is obtained. The shape and size of the internal fracture is essentially spherical whose volume ranges from a pinpoint to several millimeters in diameter. On the basis of a simple consideration of the transmission of elastic strain energy, corresponding sizes of fracture can be analyzed and estimated. Comparison of the theoretical results with experimental data indicates that the strain energy transmitted is directly proportional to the amount of explosive charge used.

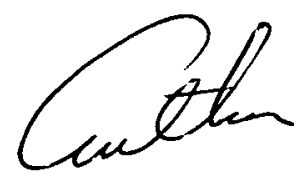

*This research work is supported in part by the U. S. AEC and NASA 
The initiation of an internal fracture at the vicinity of a point in a medium as a result of converging tensile pulses can be obtained using the geometry of a prolate spheroid. $^{l}$ This three dimensional case is more complicated than fractures observed in rods, plates, etc. by Kolsky ${ }^{2}$ and others. A prolate spheroid has the property that every ray emerging from one of its foci will be reflected from the free surface and converge to the other focus after travelling paths of equal length. Through the detonation of some high explosives at one focus, the created sharp compressive pulses will travel radially to the free surface and be reflected as relatively fast tensile pulses which converge toward the other focus, and as slower shear waves which converge along the spheroidal axis. Fracture around the second focus will occur when the magnitude of the tensile pulses becomes sufficiently large for certain lengths of time. The exact analysis of the state of stress in the neighborhood of the second focus is extremely difficult if not impossible.

The governing general equation of motion in terms of displacement can be decoupled into two sets of wave equations containing two potential functions associated with dilatational waves and shear waves. The boundary conditions are such that over the spheroidal surface both the normal and tangential stress components vanish. Using spheroidal coordinates these conditions can be expressed in terms of the potential functions. In the neighborhood of the focus where detonation occurs the material is subjected to very large compressive stresses. As a result the equations of linear elasticity may not be applied. This situation can be avoided by choosing a small sphere such that outside this sphere the compressive stresses are small enough to justify the use of linear elasticity equations. Consequently, the inner boundary conditions over the spherical surface are also describable.

1. One of the authors (CCH) is indebted to Professor Kolsky for the opportunities of discussing the subject with him while visiting at Brown University.

2. H. Kolsky, Stress Waves in Solids, Dover, 1963 
The wave equations together with these boundary conditions completely specify the problem. ${ }^{3}$ Although the wave equations can be easily separated into ordinary wave functions of zero order, ${ }^{4}$ it is extremely difficult to determine the solution satisfying all the lengthy boundary conditions. Therefore, the state of stress at a point in the spheroid at any instant cannot be determined by routine analytical methods.

For the present problem, however, considerations of the propagation, reflection and convergence of only the incident dilatation waves might give some significant information in as much as the transmission of elastic strain energy and fracture are concerned. The rate of transmission of energy across any closed surface is obtainable by integrating the general equation of motion. Further integration of the rate of transmission of strain energy over a period of time, say between $t_{1}$ and $t_{2}$, could yield the total reflected strain energy transmitted during this period to the second focus. Since all direct paths of the dilatational waves between two foci are equal, the second focus will be under uniform hydrostatic tension. Assume that a small neighborhood near the second focus is also under hydrostatic tension. If $\delta$ is the radius of the fracture and $E$ the magnitude of strain energy per unit volume of the fracture region of the medium, then the total reflected strain energy is

$$
\mathrm{W} \cong \frac{4}{3} \pi \delta^{3} \mathrm{E}
$$

or

$$
\delta \cong\left(\frac{3}{4} \frac{W}{\pi E}\right)^{1 / 3}
$$

In the case when $E$ is not known, and $W_{m}$ is the maximum

3. C. C. Hsiao, S. R. Moghe, C. K. Soong and W. L. Yin, "Internal Fracture under Radial Tension," presented at the International Conference on Fracture, Sendai, Japan, September 12-17, 1965.

4. P. M. Morse and H. Feshbach, Methods of Theoretical Physics, McGraw-Hill, 1953. 
value of the reflected strain energy and $\mathrm{W}_{\mathrm{O}}$ is the minimum value below which no fracture will occur, then for a material with Poisson ration $1 / 3$, the total strain energy which will cause fracture can be obtained as follows:

$$
W_{m}-W_{0}=\iiint_{r S \delta} \sigma_{i j} e_{i j} d V \cong \frac{\pi}{2 \mu} p^{2} \delta^{3}
$$

where the volume integral is evaluated for a sphere whose radius $r$ is less or equal to $\delta, \sigma_{i j}$ and $e_{i j}$ are respectively stress and strain tensors, $\mu$ is the shear modulus of the medium and $\mathrm{p}$ is the hydrostatic tension under which fracture occurs. Or

$$
\delta \cong\left[\frac{2 \mu}{\pi} \frac{\left(w_{m}-w_{0}\right)}{p^{2}}\right]^{I / 3} \quad ; \quad w_{m} \geq w_{0}
$$

The above relation gives a theoretical estimate on the fracture for a given $p$ and $W_{m}$. Fig. 1 gives the relationship between $W_{m}-W_{0}$ and $\delta$. It is interesting to note that for constant $W_{m}$ the larger value of $p$ the smaller will be $\delta$. With measured $\delta$, the same relation can also be used in the estimation of hydrostatic tension $p$ of fracture provided that the magnitude of strain energy can be determined first.

Experimentally by exploding lead azide at one focus of a prolate spheroid made of polystyrene or polymethyl methacrylate materials, fracture of the specimen did occur. ${ }^{5}$ Fig. 2 illustrates as indicated two kinds of fractured specimens. Fig. 3 shows a series of fractured polystyrene spheroids under different explosive charges. Fig. 4 gives the plot of the size of fracture against the amount of explosive charge used. It is of great interest to note that by comparing the curves in Fig. I and Fig. 4 on similar scales the strain energy transmitted is directly proportional to the amount of explosive charge used. Therefore, if its absolute magnitude could be determined, then the internal

5. C. C. Hsiao, "Internal Fracture under Radial Tension," Bulletin, Am. Phys. Soc., March (1964) 
stress $p$ required for fracture under a state of hydrostatic tension would be found.

It might also be of importance to mention that during the course of this investigation, the size of the spheroidal specimen was not found to contribute significant difference in the internal fracture as long as the total distance between the two foci was the same. However, when the ratio of the major to minor axis of the spheroid becomes larger than 4 , the foci are very close to the surface and fracture under the same conditions will not be easily obtained, In fact, no fracture was observed possible for spheroids having a major axis of 3 inches and minor axis $1 / 2$ inch.

\section{ACKNOWLEDGMENT}

The authors would like to acknowledge the efforts made in assisting our experimental setup and in preparing excellent spheroidal specimens by the machine shops of the Department of Aeronautics and Engineering Mechanics on our Minneapolis Campus and especially Rosemount. The drawing of the figures by T. C. Chang and the typing of the manuscript by Liv Dahl are also greatly appreciated. The assistance of the University Photographic Laboratory on our St. Paul Campus in preparing the photographs is gratefully acknowledged. 


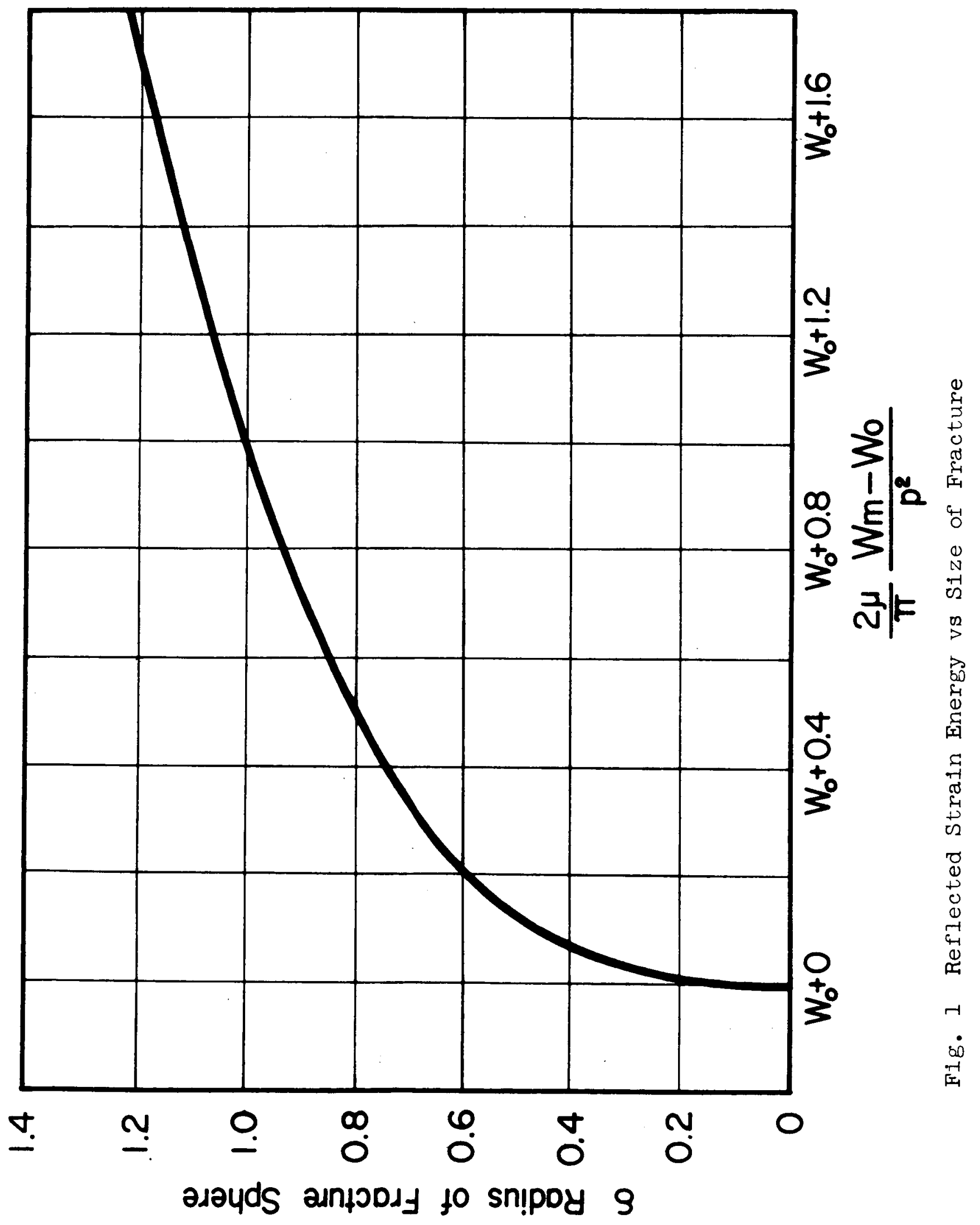




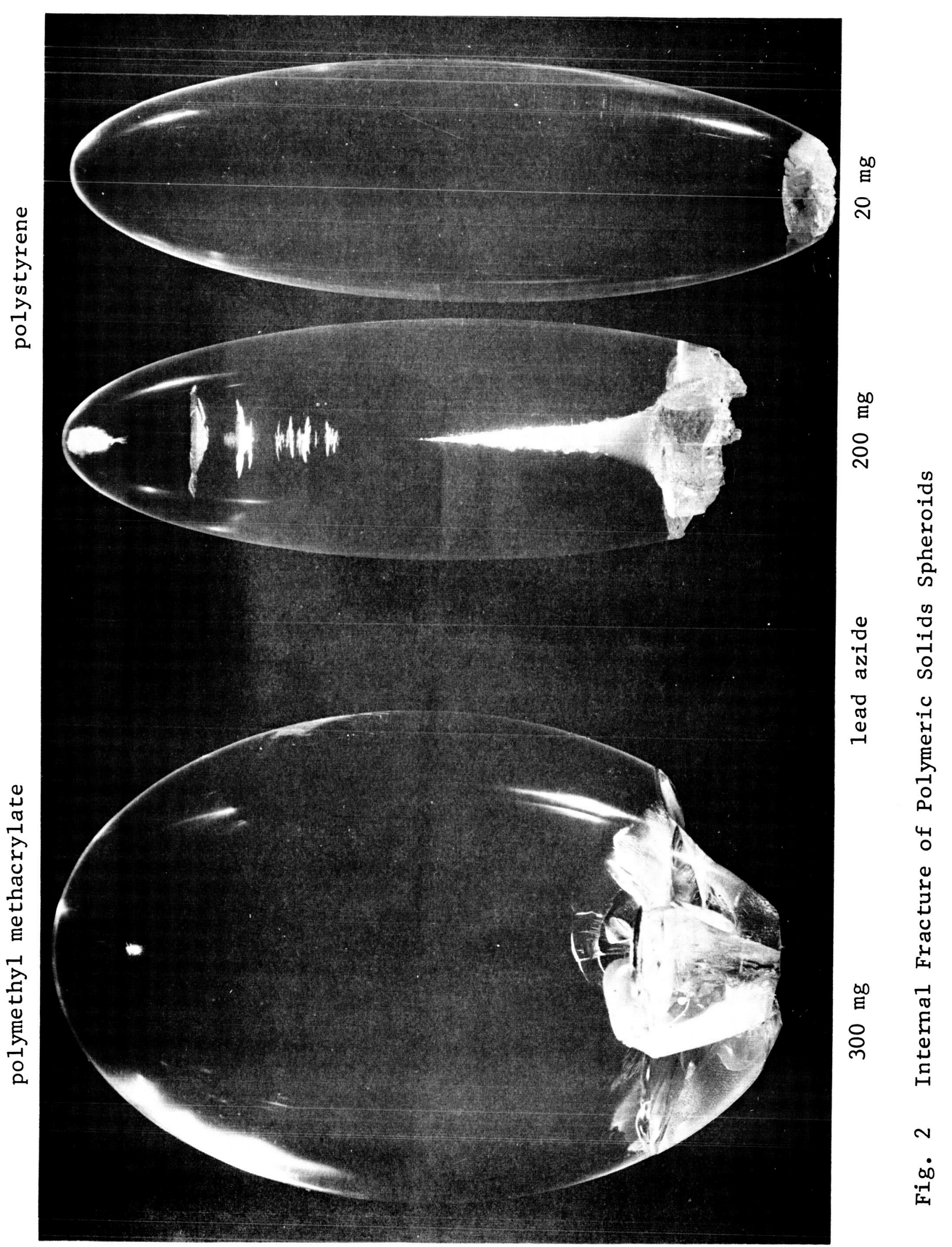




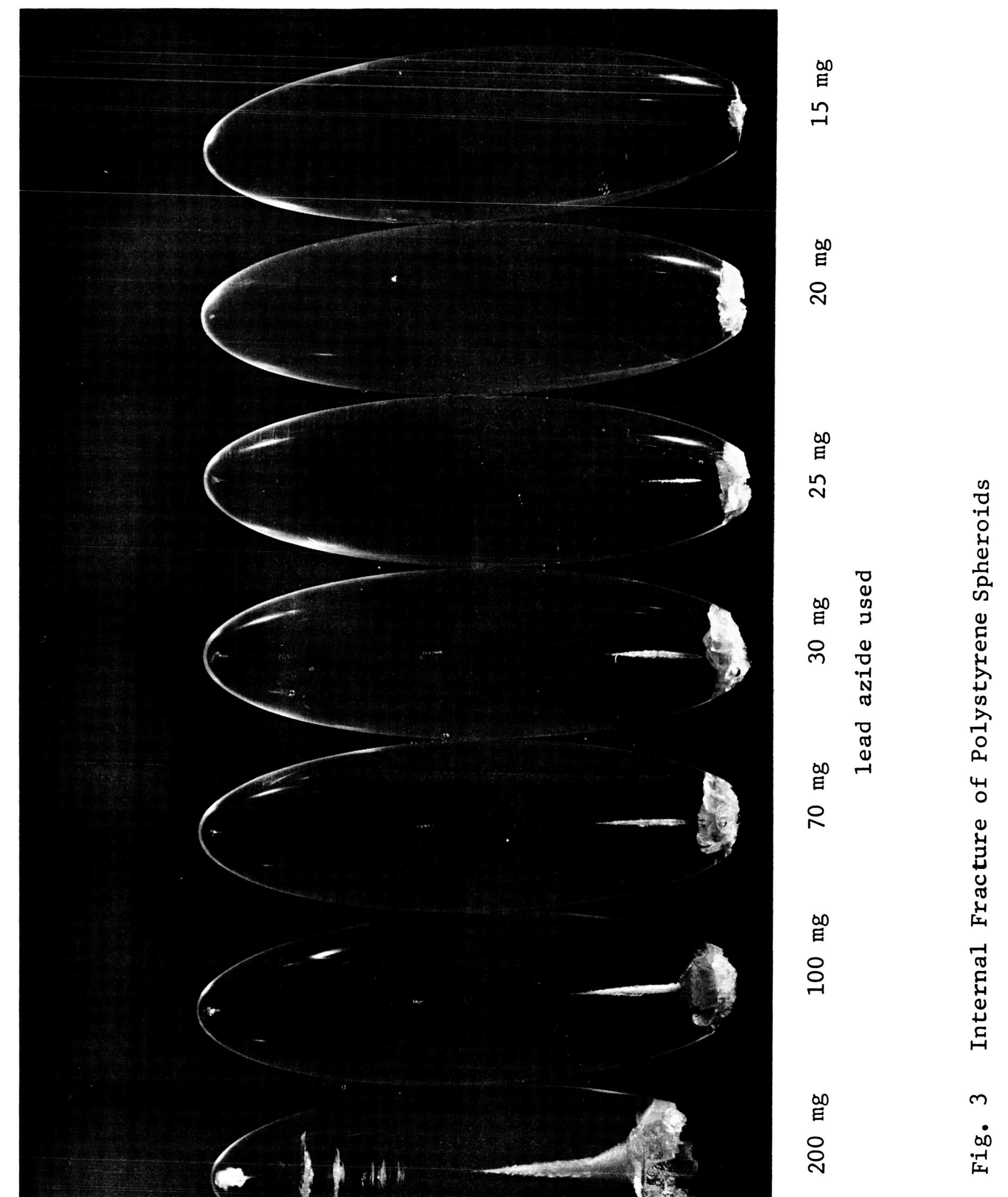




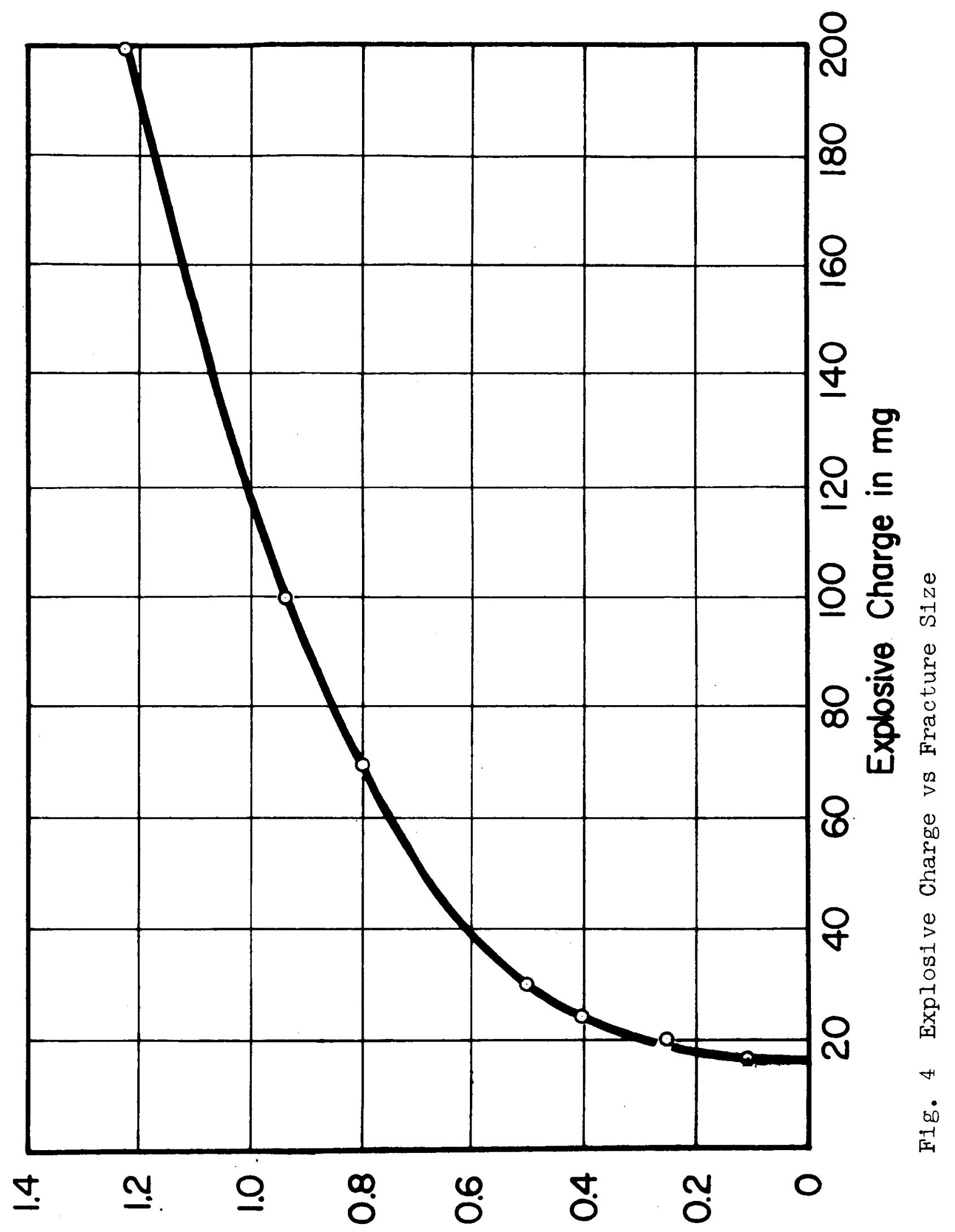

ww u! әdәyds adnłoDdy to sn!poy 